\title{
Neutron decay to a non-Abelian dark sector
}

\author{
Fatemeh Elahi® and Mojtaba Mohammadi Najafabadi \\ School of Particles and Accelerators, Institute for Research in Fundamental Sciences IPM, \\ Tehran 19395-5531, Iran
}

(Received 30 May 2020; accepted 15 July 2020; published 11 August 2020)

\begin{abstract}
According to the Standard Model (SM), we expect to find a proton for each decaying neutron. However, the experiments counting the number of decayed neutrons and produced protons disagree. This discrepancy suggests that neutrons might have an exotic decay channel to Dark Sector (DS) particles. In this paper, we explore a scenario where neutrons decay to a dark Dirac fermion $\chi$ and a non-Abelian dark gauge boson $W^{\prime}$. In our proposed scenario, the DS has three portals with the SM sector: (1) the fermion portal coming from the mixing of the neutron with $\chi,(2)$ a scalar portal, and (3) a nonrenormalizable kinetic mixing between photon and dark gauge bosons which induces a vector portal between the two sectors. We discuss the cosmological implications of this scenario assuming DS particles are produced via freeze-in. The fermion and the scalar portal leads to the overproduction of DS particles by the time of the Cosmic Microwave Background (CMB), and thus we disable these two portals in the early universe. For that, we require the maximum temperature of the universe to be lower than $m_{\gamma}$. We rely on the vector portal to connect the two sectors in the early universe, and we discuss the phenomenological bounds on the model. The main constraints come from the Big Bang Nucleosynthesis, ensuring the right relic abundance of dark matter, and the observation of large neutron stars.
\end{abstract}

DOI: 10.1103/PhysRevD.102.035011

\section{INTRODUCTION}

Even though the Standard Model (SM) of particle physics can explain almost all observed phenomena, we are certain there exists physics beyond the SM. One of the most prominent questions in the particle astrophysics community is the nature and origin of dark matter (DM). So far, we have not observed any unambiguous detection of DM. However, numerous experimental anomalies may be a hint of DM interaction with the SM. One of these experiments is the measurements of the neutron lifetime.

Due to the importance of neutrons as one of the main building blocks of luminous matter and one of the key role players in the formation of light elements in the early universe, there have been several experiments that attempt to find the lifetime of the neutrons [1-10]. In the SM, we expect the branching ratio of a neutron decaying to a proton, an electron, and a neutrino $\left(n \rightarrow p+e+\bar{\nu}_{e}\right)$ to be $100 \%$. In an experiment known as the bottle experiment [1-8], ultracold neutrons are stored for a time comparable to the neutron lifetime, then the remaining neutrons are counted. This experiment finds the total decay width or,

Published by the American Physical Society under the terms of the Creative Commons Attribution 4.0 International license. Further distribution of this work must maintain attribution to the author(s) and the published article's title, journal citation, and DOI. Funded by SCOAP ${ }^{3}$. equivalently, the lifetime of the neutrons. Their finding is $\tau_{n}^{\text {bottle }}=879.6 \pm 0.6 s$. In another experiment known as the beam experiment $[9,10]$, the number of produced protons is counted, and their finding has been announced to be $\tau_{n \rightarrow p+\cdots}^{\text {beam }}=888.0 \pm 2.0 \mathrm{~s}$. The lifetime of neutrons in these two experiments differs by $8.4 \mathrm{~s}$ with a significance of $4 \sigma$. The aforementioned discrepancy may be the result of an exotic decay of neutrons to the dark sector. Due to the close mass of neutrons and protons and their intimate structures, the easiest way to ensure an exotic decay of a neutron and the stability of protons is to assume the total mass of the exotic decay of neutron $M_{f}$ is greater than the mass of proton and electron: $m_{n}>M_{f}>m_{p}-m_{e}$. Recently, the analysis of hydrogen lifetime using Borexino data $[11,12]$ has made the lower bound on the mass of the exotic particle even stronger (e.g., $M_{f}>m_{p}+m_{e}$ ). Furthermore, baryon number violating processes are severely constrained [13-27]. Therefore, we are led to consider scenarios where neutrons can decay to a new degree of freedom that has a baryonic charge, but is heavy enough that it can subsequently decay to protons.

Numerous studies have explored different possibilities [28-48]. An important ingredient in most of these studies is a mixing between the neutron and a new fermionic degree of freedom $(\chi)$ with a baryon charge of +1 . An effective Lagrangian can be written as

$$
\mathcal{L}_{\text {eff }}=\bar{\chi}\left(i \not D-m_{\chi}\right) \chi-\bar{n}\left(i \not \partial-m_{n}+\mu_{n} \sigma^{\mu \nu} F_{\mu \nu}\right) n-\delta m \bar{n}_{R} \chi_{L},
$$


where $\mu_{n}=-0.617 \mathrm{GeV}^{-1}$ is the neutron magnetic dipole moment, and $\delta m$ is the mixing between neutron and $\chi$. To resolve the neutron lifetime discrepancy, $\delta m$ is expected to be about $10^{-13} \mathrm{MeV}$ [28]. The minimal scenario is assuming the dominant exotic decay of neutron is $n \rightarrow \chi \gamma$. If we assume $m_{p}+m_{e}<m_{\chi}<m_{n}$, we expect $E_{\gamma} \lesssim 1 \mathrm{MeV}$ [28]. Experimental measurements disfavor such decay of a neutron if the photon energy is in the range $0.782 \mathrm{MeV}<$ $E_{\gamma}<1.664 \mathrm{MeV}$ up to $2.2 \sigma$ significance [49,50], but softer photons remain unexplored. Another important constraint comes from the conversion of neutrons to $\chi$ in Neutron Stars (NSs) [30]. Specifically, if neutron and $\chi$ are in chemical equilibrium, due to the less interaction of $\chi$ comparatively, the conversion of neutrons to $\chi$ leads to a lower pressure. Integrating the Tolman-Oppenheimer-Volkoff equations $[39,41,42,51-56]$, one can find the maximum mass of a NS as a function of its radius, and the upper limit is in contradiction with the properties of some of the neutron stars observed [30]. The simplest solution is to consider DM scenarios that have repulsive self-interaction and a repulsive interaction with neutrons. That is to have a vector mediator, e.g., a dark photon.

In Ref. [30], the authors considered the decay of $n \rightarrow \chi A^{\prime}$. To ensure the theory is consistent with the observation of dense NSs with mass $2 M_{\odot}$, we need $m_{A^{\prime}} /$ $g_{D} \lesssim(45-60) \mathrm{MeV}$, where $g_{D}$ is the gauge coupling of the $U(1)_{D}$. In this setup, there is a mixing between the dark gauge boson and the SM photon:

$$
\mathcal{L}=-\frac{\epsilon}{4} F_{\mu \nu}^{\prime} F^{\mu \nu}
$$

which induces dark photon-electromagnetic current interaction with a coupling proportional to $\epsilon$. The authors of Ref. [30] did an extensive phenomenological study of this scenario, and showed that the parameter space for $m_{A^{\prime}}<$ $2 m_{e}$ is severely constrained. ${ }^{1}$ One of the main constraints comes from the era of Big Bang Nucleosynthesis (BBN), which requires dark photon to decay early enough that it does not inject much energy during $\mathrm{BBN}$. One way to loosen this constraint is to produce the dark sector (DS) particles via freeze-in. For a successful freeze-in scenario, we need a feeble coupling between DS particles and the SM sector. The couplings of $\chi$, however, are determined from explaining the neutron decay experiments. We show that the $\chi-n$ mixing with strength $10^{-13} \mathrm{MeV}$ is small enough that $\chi$ counts as a DM in the data from the Cosmic Microwave Background (CMB), and it is big enough that leads to the overproduction of $\chi$ in the early universe. To resolve this issue, we require the maximum temperature of the universe to be smaller than $m_{\chi}$ so that $\chi$ is not produced in the early universe. Therefore, we need to rely on the dark photon or the scalar to explain the DM relic abundance.

\footnotetext{
${ }^{1}$ After the Borexino constraint [11], the case where $m_{A^{\prime}}>2 m_{e}$ is less attractive.
}

However, both of which are unstable particles. Hence, in this paper, instead of a dark $U(1)_{D}$, we consider a dark non-Abelian gauge $S U(2)_{D}$. That is because even though it does not have any more free parameters, the extra degrees of freedom help with explaining the two observations of DM and neutron decay. Furthermore, for the freeze-in scenario to work, we should employ very small kinetic mixing, and this is more justified in the non-Abelian kinetic mixing because of its nonrenormalizable nature. The main differences between our work and Ref. [30] are the following:

In this paper, DS has a gauge $S U(2)_{D}$ rather than a gauge $U(1)_{D}$.

We assume the relic abundance of DS particles is through freeze-in.

We turn off the scalar portal between the two sectors. More specifically, in the potential term $\lambda_{\phi H}|\phi|^{2}|H|^{2}-$ with $\phi$ being the scalar responsible for the spontaneous breaking of the $S U(2)_{D}$ and $H$ being the SM Higgs-we take $\lambda_{\phi H}=0$. We argue that the radiative correction is very suppressed, and thus our assumption is justifiable. This choice of $\lambda_{\phi H}$ has important consequences for the relic abundance of DM.

The organization of the paper is as follows: In Sec. II we explain the model and introduce the degrees of freedom as well as the free parameters in the theory. Section III is devoted to the phenomenology of the model including the constraints from NSs, the neutron decay experiments, and the cosmological constraints which are discussed in Sec. III A. Indirect Detection is discussed in Sec. III B, Direct Detection and Collider Constraints are explored in Sec. III C, and, finally, the concluding remarks are presented in the Conclusion, Sec. IV.

\section{MODEL}

Let us assume the Dark Sector has a gauge $S U(2)_{D}$ that is spontaneously broken by a doublet $\phi$ :

$$
\phi=\left(\begin{array}{c}
\frac{1}{\sqrt{2}}\left(G_{\phi}^{1}+i G_{\phi}^{2}\right) \\
\varphi+v_{\phi}+i G_{\phi}^{3}
\end{array}\right),
$$

where $G_{\phi}^{i}$ are the Goldstone bosons, which become the longitudinal component of the gauge bosons, and $v_{\phi}$ is vacuum expectation value (vev) of $\phi$. To ensure $\phi$ indeed acquires $v e v$, we require its potential to have the following form:

$$
\begin{aligned}
V(\phi, H)= & -\mu_{\phi}^{2}|\phi|^{2}+\lambda_{\phi}|\phi|^{4}-\mu_{H}^{2}|H|^{2}+\lambda_{H}|H|^{4} \\
& +\lambda_{\phi H}|\phi|^{2}|H|^{2}
\end{aligned}
$$

with $\mu_{\phi}^{2}>0$. Since the neutron is a fermion, the decay of a neutron to DS particles compels us to include fermionic degrees of freedom. Thereby, we introduce a Dirac fermion $\chi$ transforming as a doublet under $S U(2)_{D}: \chi^{T}=\left(\chi_{1}, \chi_{2}\right)$. 
Since there are severe constraints on the baryon number violating models $[13,14]$, we assume $\chi$ has a baryon charge of +1 . In this setup, the effective Lagrangian including the new degrees of freedom becomes

$$
\begin{aligned}
\mathcal{L}_{N P}= & -\frac{1}{4} W_{\mu \nu}^{\prime a} W^{\prime a, \mu \nu}+i \bar{\chi}\left(D+m_{\chi}\right) \chi+\left|D_{\mu} \phi\right|^{2}+\eta \bar{\chi}_{L} \phi n \\
& + \text { H.c. }+C_{Y} \operatorname{Tr}\left[\phi^{\dagger} \tau^{a} \phi W_{\mu \nu}^{\prime a} F^{\mu \nu}\right] \\
& +\tilde{C}_{Y 1} \operatorname{Tr}\left[\phi^{\dagger} \tau^{a} \phi W_{\mu \nu}^{\prime a} \tilde{F}^{\mu \nu}\right] \\
& +\tilde{C}_{Y 2} \operatorname{Tr}\left[\phi^{\dagger} \tau^{a} \phi \tilde{W}_{\mu \nu}^{\prime a} F^{\mu \nu}\right] \\
& +h . c-V(\phi, H),
\end{aligned}
$$

where $W_{\mu \nu}^{\prime a}$ is the field strength tensor of $S U(2)_{D}$ and $\tilde{W}_{\mu \nu}^{\prime a}=\epsilon_{\mu \nu \alpha \beta} W^{\prime a, \alpha \beta}$ is the dual of the field tensor, and $D_{\mu}=\partial_{\mu}-i g_{D} \tau^{a} W_{\mu}^{a}$. The Lagrangian terms written in the second line of Eq. (5) are the non-Abelian kinetic mixing between the field tensor of $S U(2)_{D}$ gauge bosons and that of photon. Due to the presence of $\phi$ in these terms the kinetic mixing with the $C P$-odd component is not a total derivative, and thus contributes to the action. For simplicity, we assume $\tilde{C}_{Y} \equiv \tilde{C}_{Y 1}=\tilde{C}_{Y 2}$. Note that the kinetic mixing terms are operators of dimension 6 and therefore have an inverse mass-squared dimension. The suppressed mass dimension means there is a small coupling of the dark gauge bosons with SM particles.

After $S U(2)_{D}$ Spontaneous Symmetry Breaking (SSB), $W^{\prime}$ and $\phi$ get a mass proportional to $v_{\phi}$ :

$$
m_{W^{\prime}}=g_{D} v_{\phi} \quad m_{\phi}=\sqrt{\lambda_{\phi}} v_{\phi} .
$$

At low energies, there is a residual $\mathbb{Z}_{2}$ symmetry remaining from the broken $S U(2)_{D}$. Under the $\mathbb{Z}_{2}$ symmetry, $W^{\prime \pm}$ and $\chi_{2}$ are odd, ${ }^{2}$ and the rest of the particles are even. To explain the neutron decay anomaly $\left(n \rightarrow \chi W^{\prime \pm}\right)$ and yet be safe from the NS constraint, we necessarily need to have $m_{\chi} \gg m_{W^{\prime}}$. Therefore, $W^{ \pm}$are the lightest particles charged under $\mathbb{Z}_{2}$, and thus are stable.

This is while $W_{3}^{\prime}$ mixes with photon after $\phi$ gets a vev, and thus it can decay (e.g., $W_{3}^{\prime} \rightarrow 3 \gamma$ ). Similarly, due to the $\phi W_{3}^{\prime} W_{3}^{\prime}$ coupling we can have $\phi \rightarrow \gamma \gamma$ decay. It is worth mentioning that $\chi, W_{3}^{\prime}$, and/or $\phi$ could be long-lived DM candidates.

The free parameters in this model are the following:

(i) masses: $m_{\chi}, m_{\phi}, v_{\phi}$.

(ii) couplings: $\eta, g_{D}, \lambda_{\phi H}$, and $C_{Y}, \tilde{C}_{Y}$ with dimensions proportional to $\left[\mathrm{M}^{-2}\right]$.

To find the cosmological constraints on the model, we need to briefly discuss the UV completion of the model.

\footnotetext{
${ }^{2}$ Even though after $\phi$ acquires a vev, there is a slight mass splitting between $\chi_{1}$ and $\chi_{2}$, this mass splitting is negligible compared with $m_{\chi}$. Therefore, for the rest of the paper, we will assume both $\chi_{1}$ and $\chi_{2}$ have mass $m_{\chi}$ and we will use $\chi$ to refer to both of them.
}

This is very similar to the model suggested in Ref. [30]: two color triplet scalars with hypercharge $1 / 3\left(\Phi_{1}\right.$ and $\left.\Phi_{2}\right)$ are introduced, where $\Phi_{1}$ is also a doublet of $S U(2)_{D}$. Thus, the UV Lagrangian can simply be written as

$$
\mathcal{L}_{\mathrm{UV}}^{n-\chi}=\lambda_{1} \bar{d}^{a} P_{L} \chi \Phi_{1 a}+\lambda_{2} \epsilon^{a b c} \bar{u}_{a}^{c} P_{R} d_{b} \Phi_{2 c}+\mu_{1} \Phi_{1 a} \Phi_{2}^{* a} \phi
$$

where in the effective theory

$$
\eta=\frac{\beta v_{\phi} \lambda_{1} \lambda_{2}}{m_{\Phi_{1}}^{2} m_{\Phi_{2}}^{2}}
$$

with $\beta$ being the factor derived from confinement of quarks to neutrons, and its value $\beta=0.014 \mathrm{GeV}^{3}$ is taken from Lattice QCD simulations [57]. Dijet searches at CMS [58] and ATLAS [59] push the masses of $\phi_{i}$ to greater than $1 \mathrm{TeV}$.

Since $\Phi_{i}$, with $i=1,2$ are charged under $S U(3)_{c}$, we expect their number density in the early universe to match that of photons (e.g., we expect them to be in thermal equilibrium with thermal bath). Through their couplings with the dark sector, the production of $\chi$ and subsequently $\phi$ and $W^{\prime}$ should occur in abundance. As shown in [30], such set up leads to severe constraints from CMB [60-62], BBN [63], and the Fermi-LAT observation of gamma rays from dwarf spheroidal galaxies $[64,65]$. The summary of these constraints is presented here:

Once $\chi$ becomes nonrelativistic, it can only annihilate to $W^{\prime} W^{\prime}$ and $\phi \phi$ efficiently. Therefore, if $\chi$ is in thermal equilibrium in the early universe, the abundant production of $W^{\prime}$ and $\phi$ becomes inevitable. On the other hand, $W_{3}^{\prime}$ and $\phi$ can only decay after $\phi$ acquires a $v e v$, which roughly occurs around $60 \mathrm{MeV}^{3}$, and it is extremely close to BBN. The decay of $W_{3}^{\prime}$ and $\phi$ near the BBN disturbs the Hubble rate and thus it significantly alters the production of light nuclei by diluting the baryon-photon ratio as well as causing photodissociation of the nuclei.

Similarly, decays near and during recombination will distort the CMB temperature fluctuations and thus there are severe constraints on a model with light $W^{\prime}$ from CMB as well.

For $m_{W^{\prime}} \ll m_{\chi}$, the annihilation of $\chi \chi$ to $W^{\prime} W^{\prime}$ is Sommerfeld enhanced at low velocities, which leads to an enhanced annihilation cross-section in spheroidal galaxies and at the time of recombination. Therefore, it is crucial that we do not have much $\chi$ in the universe.

Due to the hydrogen lifetime constraint derived recently by [11], a $\chi$ that explains neutron lifetime discrepancy

\footnotetext{
${ }^{3}$ This value is the maximum value allowed from the NS constraint.
} 


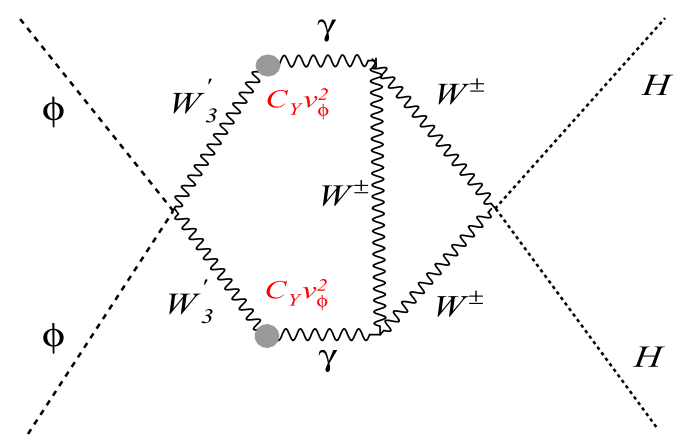

FIG. 1. The radiative corrections to $\lambda_{\phi H}$. As illustrated this coupling is suppressed by two loops as well as $C_{Y}^{2} v_{\phi}^{4}$, and thus it is very small. Therefore, if we let $\lambda_{\phi H}=0$, we can easily make sure the radiative corrections to $\lambda_{\phi H}$ stay negligible.

will need to be heavier than $m_{p}+m_{e}$. Therefore, the mass of dark gauge boson needs to be smaller than $2 m_{e}$. As discussed in Ref. [60], this case with a freezeout mechanism is completely ruled out.

Thereby, in this paper, we explore another avenue. We assume dark sector particles start with zero abundance in the early universe and they get produced through the freezein mechanism [66-72]. Consequently, in our setup, we need the maximum temperature $T_{\max }$ to be smaller than $m_{\Phi_{i}}$ so that they are not produced in the early universe. If the color multiplets are not produced, then the production of $\chi$ is greatly reduced.

The portals between the dark sector and the SM sector are via (1) the Higgs portal with a strength proportional to $\lambda_{\phi H}$, (2) the kinetic mixing governed by $C_{Y} v_{\phi}^{2}$, and (3) the effective mixing between $\chi$ and neutron which is $\eta v_{\phi}$. For a successful freeze-in scenario, we need an extremely weak connection between the dark sector and the SM sector. Since $C_{Y}$ and $\eta$ are due to nonrenormalizable interactions, we can justifiably assign them small values. ${ }^{4}$ This argument becomes more nontrivial for $\lambda_{\phi H}$, which in general can take any value $\lesssim 1$. If we want to assign $\lambda_{\phi H}$ a small value, we must make sure that this choice is safe from loop corrections. The radiative correction to $\lambda_{\phi H}$ comes from $\phi \phi \gamma \gamma$ vertex, which is suppressed by $g_{D} C_{Y}^{2} v_{\phi}^{4}$. Figure 1 shows one of the leading diagrams to radiative correction to $\lambda_{\phi H}$, and, as it is illustrated, in addition to the $C_{Y}^{2} v_{\phi}^{4}$ suppression, it is two loops suppressed. Therefore, if the value of $\lambda_{\phi H}$ is small at tree level, it does not get amplified significantly at loop levels. For simplicity, in this work, we assume $\lambda_{\phi H}=0$.

Having closed the Higgs portal, now we need to discuss the evolution of dark sector particles in the early universe, and how much they contribute to the relic abundance of the total DM. In the following section, we discuss the

\footnotetext{
${ }^{4}$ The value of $\eta$ is governed by the neutron decay anomaly. In Sec. III A 1, we show that the values of $\eta$ in all of our benchmarks are too big for a successful freeze-in scenario.
}

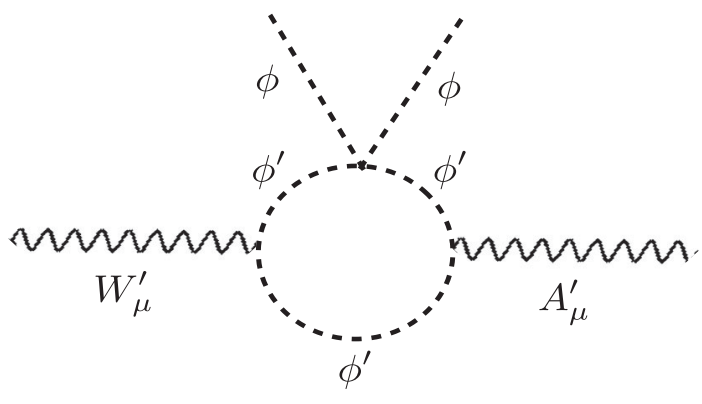

FIG. 2. A possible UV completion to the non-Abelian kinetic mixing terms is presented in this diagram, where in the effective theory the particle $\phi^{\prime}$ has been integrated out. In this theory, the particle $\phi^{\prime}$ is a doublet of $S U(2)_{D}$ and has a nonzero hypercharge (or equivalently a nonzero electromagnetic charge).

phenomenological constraints on each of these parameters including the ideal spot that explains the neutron decay anomaly and yields the correct relic abundance of DM. Even though the number of free degrees of parameters is large, the numerous experimental and observations bounds on these parameters forces us to live in a small region of the parameter space.

\section{A. Aside}

The UV Lagrangian presented in Eq. (6) only describes the neutron- $\chi$ mixing. The dimension six operators in Eq. (5) cannot be UV completed with the degrees of freedom in Eq. (6). The UV completion of Eq. (5) can be done in several ways. For example, one or a combination of the following theories can generate the non-Abelian kinetic mixing terms:

(1) Assume there exists $\phi^{\prime}$, which is a doublet of $S U(2)_{D}$ and charged under $U(1)_{\mathrm{EM}}$. The UV Lagrangian in this case contains

$$
\mathcal{L}_{\mathrm{UV}_{1}} \supset\left|D_{\mu} \phi^{\prime}\right|^{2}+\lambda_{\phi^{\prime} \phi}^{\prime}\left(\phi^{\prime \dagger} \phi\right)\left(\phi^{\dagger} \phi^{\prime}\right)
$$

where $D_{\mu}=\partial_{\mu}-i g_{D} \tau^{a} W_{\mu}^{a}-i q_{\mathrm{EM}} A_{\mu}$. Through these terms, we can generate the nonrenormalizable terms through Fig. 2.

(2) Another case is to assume in the UV, there are vectorlike fermions $F_{1}$ and $F_{2}$. If $F_{1}$ is a doublet of $S U(2)_{D}$ and $F_{2}$ is a singlet, and both $F_{1}$ and $F_{2}$ are charged $q_{\mathrm{EM}}$ under $U(1)_{\mathrm{EM}}$, then the corresponding Lagrangian becomes

$$
\begin{aligned}
\mathcal{L}_{\mathrm{UV}_{2}} \supset & \bar{F}_{1}\left(i D_{\mu}^{F_{1}}+m_{F_{1}}\right) F_{1}+F_{2}\left(i D_{\mu}^{F_{2}}+m_{F_{2}}\right) F_{2} \\
& +y_{F} \bar{F}_{1} \phi F_{2},
\end{aligned}
$$

where $D_{\mu}^{F_{1}}=\partial_{\mu}-i g_{D} \tau^{a} W_{\mu}^{a}-i q_{\mathrm{EM}} A_{\mu}$ and $D_{\mu}^{F_{1}}=$ $\partial_{\mu}-i q_{\mathrm{EM}} A_{\mu}$. Similarly, the aforementioned terms in Eq. (5) can be generated through a loop diagram, presented in Fig. 3. 


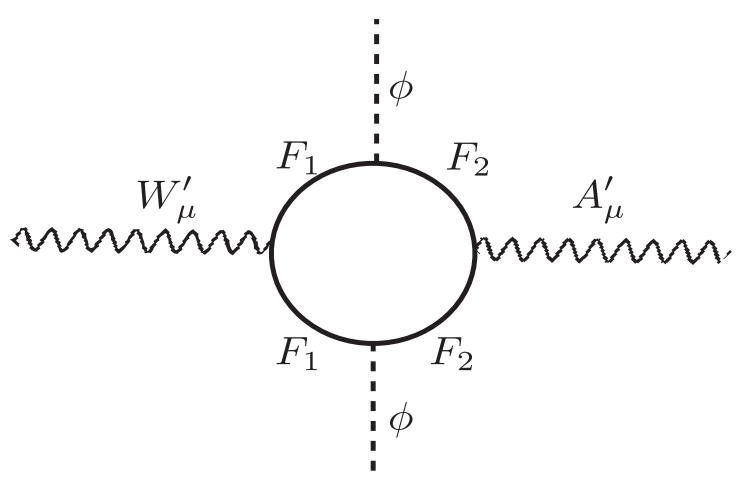

FIG. 3. Another possible UV completion to the non-Abelian kinetic mixing terms is presented in this diagram, where in the effective theory the particles $F_{1}$ and $F_{2}$ have been integrated out. In this model, $F_{1}$ and $F_{2}$ are vectorlike fermions to avoid the generation of anomalies, and they carry the same nonzero electromagnetic charge. The fermion $F_{1}$ is a doublet of $S U(2)_{D}$ and $F_{1}$ is a singlet of $S U(2)_{D}$.

\section{PHENOMENOLOGY}

One of the most important bounds on this model comes from NSs, where the conversion of the neutron to $\chi$ can have significant consequences. If neutrons and $\chi$ are in chemical equilibrium, it is favorable for the neutrons to convert into $\chi$, which, due to its almost noninteracting nature, results in a lower pressure in the neutron stars. By integrating the Tolman-Oppenheimer-Volkoff equation, one finds the maximum mass (given the NS's radius), and it falls below the largest observed mass. Our scenario fits in the category that there is a repulsive interaction between $\chi$ and the neutrons and thus can be safe from this constraint as long as $m_{W^{\prime}} / g_{D}<60 \mathrm{MeV}$ [30,51].

Another important restriction in this model comes from neutron decay. As it has been shown in Refs. [30,49], the decay of neutron ${ }^{5}$ to $\chi W^{\prime}$ and $\chi \phi$, with the diagrams shown in Fig. 4, are

$\Gamma_{n \rightarrow \chi W^{\prime}} \simeq \frac{\eta^{2}}{8 \pi} \frac{\sqrt{\left(m_{n}^{2}-\left(m_{\chi}+m_{W^{\prime}}\right)^{2}\right)\left(m_{n}^{2}-\left(m_{\chi}-m_{W^{\prime}}\right)^{2}\right)}}{m_{n}}$

$\Gamma_{n \rightarrow \chi \phi} \simeq \frac{\eta^{2}}{16 \pi} \frac{\sqrt{\left(m_{n}^{2}-\left(m_{\chi}+m_{\phi}\right)^{2}\right)\left(m_{n}^{2}-\left(m_{\chi}-m_{\phi}\right)^{2}\right)}}{m_{n}}$.

We have already discussed that the mass of $\chi$ should satisfy $m_{p}+m_{e}<m_{\chi}<m_{n}$, in order to both satisfy the neutron decay and yet be safe from recent hydrogen lifetime bound

\footnotetext{
${ }^{5}$ The decay of $n \rightarrow \chi \gamma$ can occur via $\bar{n} \sigma_{\mu \nu} \phi^{\dagger} \chi F^{\mu \nu}$. However, because of the null search for monochromatic photon [49] and exacerbating the tension of the axial coupling of the neutron [73], we expect this coupling to be very small and negligible in this study.
}

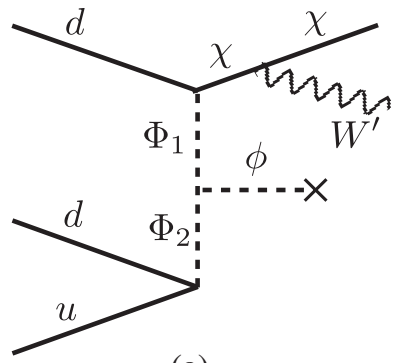

(a)

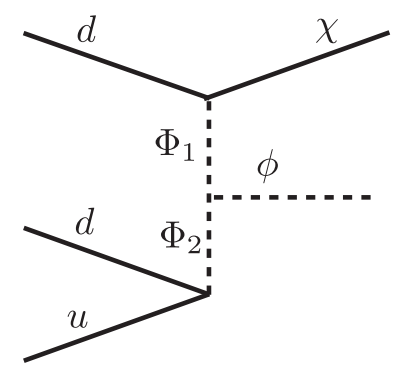

(b)
FIG. 4. The Feynman diagrams of the decay of neutron to $\chi W^{\prime}$ (a) and $\chi \phi$ (b) are presented. In these diagrams, we have shown the degrees of freedom in the UV theory that leads to the neutron$\chi$ mixing.

$[11,12]$. To have a decay that is kinematically allowed, we must have $m_{\chi}+\operatorname{Min}\left[m_{W^{\prime}}, m_{\phi}\right]<m_{n}$. Therefore, let us consider the following benchmarks:

(i) We consider two benchmarks where both $W^{\prime}$ and $\phi$ are light enough that both decays mentioned in Eq. (11) are allowed. For one of these benchmarks, we take $m_{\phi}>2 m_{W^{\prime}}$ :

$$
\begin{aligned}
m_{\chi} & =938.6 \mathrm{MeV}, \quad m_{\phi}=0.7 \mathrm{MeV}, \quad \text { and } \\
m_{W^{\prime}} & =0.2 \mathrm{MeV} .
\end{aligned}
$$

Note that in this benchmark, $\phi$ decays to $W^{\prime}$, and thus it is not a DM candidate. We will denote this benchmark as A1. To justify the neutron decay discrepancy, we need $\eta \simeq 2.7 \times 10^{-10}$.

Another benchmark we choose is when $m_{\phi} \sim m_{W^{\prime}}$ :

$m_{\chi}=938.6 \mathrm{MeV}, \quad m_{\phi}=0.7 \mathrm{MeV}, \quad$ and

$m_{W^{\prime}}=0.7 \mathrm{MeV}$,

and we present this benchmark by A2. The $\eta$ that explains the neutron decay is $\eta \simeq 2.6 \times 10^{-10}$. It is worth mentioning that $\phi$, in this benchmark, can decay to two photons. However, depending on $C_{Y}, \phi$ can be a long-lived DM candidate.

(ii) Another scenario is when $\phi$ is heavy such that the decay $n \rightarrow \chi \phi$ is not kinematically allowed. In this case, we will also take two different benchmarks; one where $m_{\phi}>2 m_{W^{\prime}}$ :

$$
\begin{aligned}
m_{\chi} & =938.6 \mathrm{MeV}, \quad m_{\phi}=2 \mathrm{MeV}, \quad \text { and } \\
m_{W^{\prime}} & =0.7 \mathrm{MeV},
\end{aligned}
$$

which we use B1 to refer to this benchmark. Solving for the $\eta$ that yields $\operatorname{Br}\left(n \rightarrow \chi W^{\prime}\right) \simeq 1 \%$ is $\eta \simeq 3.1 \times 10^{-10}$.

Another benchmark, satisfies $m_{W^{\prime}}<m_{\phi}<2 m_{W^{\prime}}$ :

$$
\begin{aligned}
m_{\chi} & =938.6 \mathrm{MeV}, \quad m_{\phi}=1.2 \mathrm{MeV}, \quad \text { and } \\
m_{W^{\prime}} & =0.7 \mathrm{MeV} .
\end{aligned}
$$


This benchmark is presented by B2. Since the only parameter that has changed is $m_{\phi}$ and $n \rightarrow \chi \phi$ is forbidden, the desired $\eta$ is still $\eta \simeq 3.1 \times 10^{-10}$.

(iii) We also consider another case where $m_{W^{\prime}}$ is large enough that the decay of $n \rightarrow \chi W^{\prime}$ is not allowed. However, $\phi$ is light enough that allows the dark decay of neutrons:

$$
\begin{aligned}
m_{\chi} & =938.6 \mathrm{MeV}, \quad m_{\phi}=0.7 \mathrm{MeV}, \quad \text { and } \\
m_{W^{\prime}} & =2 \mathrm{MeV} .
\end{aligned}
$$

This benchmark is referred by $\mathbf{C}$. The desired $\eta$ to justify the neutron decay anomaly is $\eta \simeq 4.4 \times 10^{-10}$.

\section{A. Cosmology}

In this section, we will discuss the cosmological constraints, and we will see BBN, satisfying the relic abundance give the most stringent bounds. As mentioned earlier, the observation of large neutron stars excludes part of the parameter space as well, and this constraint becomes important for heavier $W^{\prime}$.

\section{Relic abundance}

We are interested in a scenario where dark sector particles start with zero or negligible abundance and then are slowly produced through their feeble interactions with SM particles. First, we will discuss the production of $\chi$ as it will be important to set the maximum temperature of the universe, then we will investigate the evolution of $W^{\prime}$ and $\phi$ in the early universe.

\section{a. $\chi$ production}

Once $\phi$ acquires vev, a mixing between the neutron and $\chi$ is induced, which results in the conversion of neutrons to $\chi$. Through the $\chi-n$ mixing and the SM Lagrangian term $i g \bar{n} \pi^{-} \gamma_{5} p$, an interaction between $\chi-\pi-p$ is induced. Since we assume $m_{p}+m_{e}<m_{\chi}$ to be safe from hydrogen lifetime constraints [11], the decay $\chi \rightarrow p e^{-} \nu_{e}$ is kinematically allowed. However, a simple estimation of $\chi$ width yields

$$
\begin{aligned}
\Gamma_{\chi} \simeq & \frac{\eta^{2} v_{\phi}^{2}}{4\left(m_{n}-m_{\chi}\right)^{2}} \Gamma_{n \rightarrow p e \nu_{e}}\left[\left(\frac{m_{\chi}}{m_{n}}\right)^{3} \frac{1-\left(\left(m_{p}+m_{e}\right) / m_{\chi}\right)^{2}}{1-\left(\left(m_{p}+m_{e}\right) / m_{n}\right)^{2}}\right. \\
& \left.\times\left(\frac{1-\left(m_{e} /\left(m_{\chi}-m_{p}\right)\right)^{2}}{1-\left(m_{e} /\left(m_{n}-m_{p}\right)\right)^{2}}\right)^{3 / 2}\right] \\
& <\frac{\eta^{2} v_{\phi}^{2}}{4\left(m_{n}-m_{\chi}\right)^{2}} \Gamma_{n \rightarrow p e \nu_{e}},
\end{aligned}
$$

where the square bracket is the leading order estimation of the phase suppression. In our benchmarks, we have $\left(m_{n}-m_{\chi}\right)=1 \mathrm{MeV}, v_{\phi}=60 \mathrm{MeV}$, and $\eta \simeq 10^{-9}-10^{-10}$. Therefore, in all of our benchmarks, $\Gamma_{\chi}<10^{-15} \Gamma_{n \rightarrow \text { peve }}$. In other words, $\tau_{\chi}>5 \times 10^{15}$ seconds, which makes $\chi$ a longlived particle up to the time of CMB.
Up until $\phi$ acquires a $v e v,{ }^{6}$ the production of $\chi$ is due to $q q \rightarrow q \phi \chi$, where $q=u, d$. The Boltzmann equation describing the evolution of $\chi$ number is

$$
\begin{aligned}
\dot{n}_{\chi}+3 H n_{\chi}= & \int d \Pi_{q} d \Pi_{q} d \Pi_{q} d \Pi_{\phi} d \Pi_{\chi}(2 \pi)^{4} \\
& \times\left(p_{\mathrm{i}}-p_{\mathrm{f}}\right)|\mathcal{M}|_{q q \rightarrow q \phi \chi}^{2} f_{q} f_{q},
\end{aligned}
$$

where $d \Pi_{i}=\frac{d^{3} p_{i}}{(2 \pi)^{3} 2 E_{i}}$, and $f_{q} \sim e^{-E_{q} / T}$ is the distribution function of the quarks in thermal bath, and $s$ is the canonical Mandelstam variable. We can simplify Eq. (13) for any process that has three final state particles [67]:

$\dot{n}_{\chi}+3 H n_{\chi}=\frac{T}{(4 \pi)^{6}} \int d \Omega \int_{0}^{\infty} d s s^{3 / 2}|\mathcal{M}|_{q q \rightarrow q \phi \chi}^{2} K_{1}\left(\frac{\sqrt{s}}{T}\right)$,

where $K_{1}(x)$ denotes the modified Bessel function of the second kind. Equation (14) is in the relativistic limit where the masses of the particles involved are negligible to the temperature. The squared Matrix Element (ME) of $q q \rightarrow q \phi \chi$ in the relativistic limit is

$$
|\mathcal{M}|_{q q \rightarrow q \phi \chi}^{2} \simeq 36\left(\frac{\eta}{\beta}\right)^{2} s^{2} .
$$

The integral over $s$ in Eq. (14) has a closed form

$$
\int_{0}^{\infty} d s s^{(2 n+1) / 2} K_{1}\left(\frac{\sqrt{s}}{T}\right)=4^{n+1} T^{2 n+3} \Gamma(n+1) \Gamma(n+2),
$$

for $n>-1$. Therefore, we can easily calculate the right-hand side of Eq. (13). The left-hand side can be converted to yield $(Y \equiv n / S)$ with $S$ being the entropy density:

$$
\begin{aligned}
Y_{\chi}= & \int_{0}^{T_{\max }} d T\left\{-\frac{1}{S H T}\left[\frac{T}{(4 \pi)^{6}} \int d \Omega\right.\right. \\
& \left.\left.\times \int_{0}^{\infty} d s s^{3 / 2}|\mathcal{M}|_{q q \rightarrow q \phi \chi}^{2} K_{1}\left(\frac{\sqrt{s}}{T}\right)\right]\right\} \\
\simeq & \frac{3^{5} * 5^{2}}{1.66 \times \pi^{9} \sqrt{g_{\star}^{\rho}} g_{\star}^{S}} M_{\mathrm{Pl}}\left(\frac{\eta}{\beta}\right)^{2} T_{\max }^{5} \times \theta\left(T_{\max }-m_{\chi}\right) \\
\simeq & \left\{\begin{array}{ll}
3.64 & \mathbf{A 1} \\
5.1 & \mathbf{A 2} \\
7.22 & \mathbf{B 1} \& \mathbf{B 2} \\
14.55 & \mathbf{C}
\end{array} \times\left(\frac{T_{\max }}{\mathrm{GeV}}\right)^{5} \times \theta\left(T_{\max }-m_{\chi}\right),\right.
\end{aligned}
$$

\footnotetext{
${ }^{6} \mathrm{We}$ will assume that the temperature at which $\phi$ gets a vev is the value of vev itself $T_{v_{\phi}} \sim 60 \mathrm{MeV}$.
} 
where the second equality is obtained by using the definitions $S=\frac{2 \pi^{2} g_{\star}^{S} T^{3}}{45}$ and $H=\frac{1.66 \sqrt{g_{*}^{\rho}} T^{2}}{M_{\mathrm{Pl}}}$, and $\theta(x)$ is the step function that ensures the universe has enough energy to produce $\chi$. There is a constraint on the $Y_{\chi}$ so that it does not overclose the universe:

$$
Y_{\chi} \leq \frac{\Omega_{\mathrm{total} \mathrm{DM}} \rho_{c}}{m_{\chi} s_{0}} \simeq 6.6 \times 10^{-11} \times\left(\frac{\mathrm{GeV}}{m_{\chi}}\right)
$$

where for all of our benchmarks, the mass of $\chi$ is fixed to $m_{\chi}=938.6 \mathrm{MeV}$. It is clear that if $\chi$ is produced, it quickly overcloses the universe. Thereby, we require $T_{\max }<m_{\chi}$ to prevent the production of $\chi$ in the early universe. ${ }^{7}$ In other words, even though $\chi$ is a long-lived particle, it does not contribute to the relic abundance of the DM in the universe. Notice that these calculations only depend on the coupling of $\chi$ with quarks; thus, the results are the same if we had assumed a $U(1)_{D}$ instead of the $S U(2)_{D}$.

\section{b. $W^{\prime}$ and $\phi$ production}

For the case where $\lambda_{\phi H}=0$, the main mechanism for the production of $\phi$ and $W_{\mu}^{\prime}$ is via the kinetic mixing term. The leading diagram of $W^{\prime} / \phi$ production for $T>v_{\phi}$ is shown in Fig. 5, where $J_{\mathrm{EM}}$ represents any particle (lepton or hadron) that has electromagnetic charge and has a significant abundance at $T<m_{\chi}$. The Boltzmann equation governing the number density of $W^{\prime}$ and $\phi$ is similar to Eq. (14), and the squared ME for the process of our interest $J_{\mathrm{EM}} J_{\mathrm{EM}} \rightarrow \phi \phi W^{\prime}$ is the following:

$|\mathcal{M}|_{J_{\mathrm{EM}} J_{\mathrm{EM}} \rightarrow \phi \phi W^{\prime}}^{2}=\frac{Q^{4} C_{Y}^{2}\left(4 s^{2}-5 s(t+u)-5 t u\right)}{4 s}$,

with $Q$ being charge of the initial state particles, and $s, t$, and $u$ are the Mandelstam variables. In the limit where $s \gg t, u$, we get $|\mathcal{M}|_{J_{\mathrm{EM}} J_{\mathrm{EM}} \rightarrow \phi \phi W^{\prime}}^{2} \rightarrow Q^{4} C_{Y}^{2} s$. In Eq. (19), we have let $m_{W^{\prime}}=m_{\phi}=0$, because $\phi$ still has not acquired a vev. Note that $W^{\prime}$ and $\phi$ do not get thermal corrections as well, since they live in a much colder sector. In the SM sector, however, for $T \gg m_{\mathrm{SM}}(T=0)$, the thermal correction to the mass of particles becomes important. For simplicity, we assume that $m_{\mathrm{SM}}(T) \sim T$. The only exception is for proton, where for $T<m_{p}$, we assume $m_{p}(T)=m_{p}(T=0)$. Furthermore, we make the reasonable assumption that the SM unstable particles decay more efficiently to SM particles than annihilate to $W^{\prime}$ and $\phi$. The yield of $W^{\prime}$ and $\phi$ coming from Fig. 5 is

\footnotetext{
${ }^{7}$ We have not observed any evidence before the epoch of BBN. Therefore, as long as $T_{\max } \gtrsim 10 \mathrm{MeV}$, low $T_{\max }$ does not violate any cosmological observations [74].
}

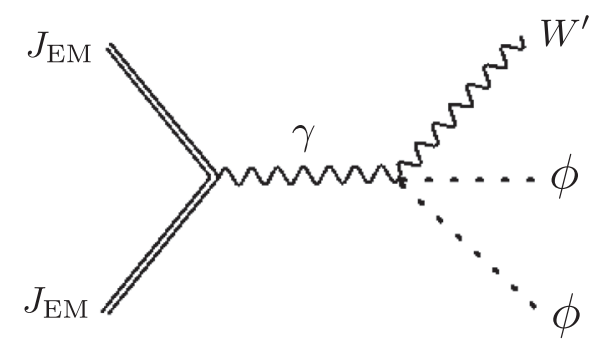

FIG. 5. The Feynman diagram that leads to the production of $\phi$ and $W^{\prime}$, where $J_{\mathrm{EM}}$ means the electromagnetic current.

$$
Y_{U V_{i}} \simeq g_{i} \alpha M_{\mathrm{Pl}} \frac{3^{3} 5^{2} \times C_{Y}^{2}}{1.66 \times 2^{4} \pi^{8} \sqrt{g_{\star}^{\rho}} g_{\star}^{S}} T_{\max }^{3},
$$

where $i=W^{\prime} / \phi$ and $g_{i}$ represents the number of $\phi$ and $W^{\prime}$ produced.

Once $\phi$ acquires a vev, the production of $W_{3}^{\prime}$ can occur through renormalizable and nonrenormalizable operators, shown in Fig. 6. The renormalizable interaction that results in the production of $W^{\prime \pm}$ is illustrated in Fig. 7. For processes with two body final states, Eq. (13) simplifies to [67]:

$$
\begin{aligned}
\dot{n}_{i}+3 H n_{i}= & g_{i} \frac{3 T}{(4 \pi)^{4}} \int d \Omega \int_{m_{\text {final }}^{2}}^{v_{\phi}^{2}} d s\left(\frac{s-m_{\text {final }}^{2}}{\sqrt{s}}\right) \\
& \times|\mathcal{M}|^{2} K_{1}\left(\frac{\sqrt{s}}{T}\right),
\end{aligned}
$$

where $m_{\text {final }}$ is the mass of the final state particles in each interaction. The exact value of the squared matrix elements as well as the approximate yield of $W_{3}^{\prime}, W^{\prime \pm}$, and $\phi$ can be found in Appendix. Depending on the benchmark, we can either have both $W^{\prime \pm}$ and $\phi$, or only $W^{\prime \pm}$ as our DM particles. However, since the production of $\phi$ and $W^{\prime \pm}$ are through similar diagrams, these two cases only differ by an $O(1)$ factor. The region that produces too much DM (i.e., $\Omega_{W^{\prime}}+\Omega_{\phi}>\Omega_{\text {total DM }}$ ) is shown in orange in Fig. 9. Even though $\phi$ is long-lived in some regions of the parameter space and can contribute to the relic abundance of DM, this fact does not affect the computations in a significant way. That is because the main production of $\phi$ and $W^{\prime}$ is due to Eq. (20). Therefore, the bounds are not very sensitive to $m_{\phi}$.

\section{2. $C M B$ and $B B N$ constraints}

We know that $W_{3}^{\prime}$ decays, and if it injects energy during $\mathrm{BBN}$, its energetic decay products might disturb the production of the light nuclei by diluting the ratio of baryons to photons. Furthermore, the injection of energy may cause photodissociation, which will affect the Cosmic Microwave Background (CMB) fluctuations. To avoid

\footnotetext{
${ }^{8}$ The small differences between Eqs. (14) and (21) are due to the number of final state particles, and the fact that final state particles are massive after $S U(2)_{D} \mathrm{SSB}$.
} 

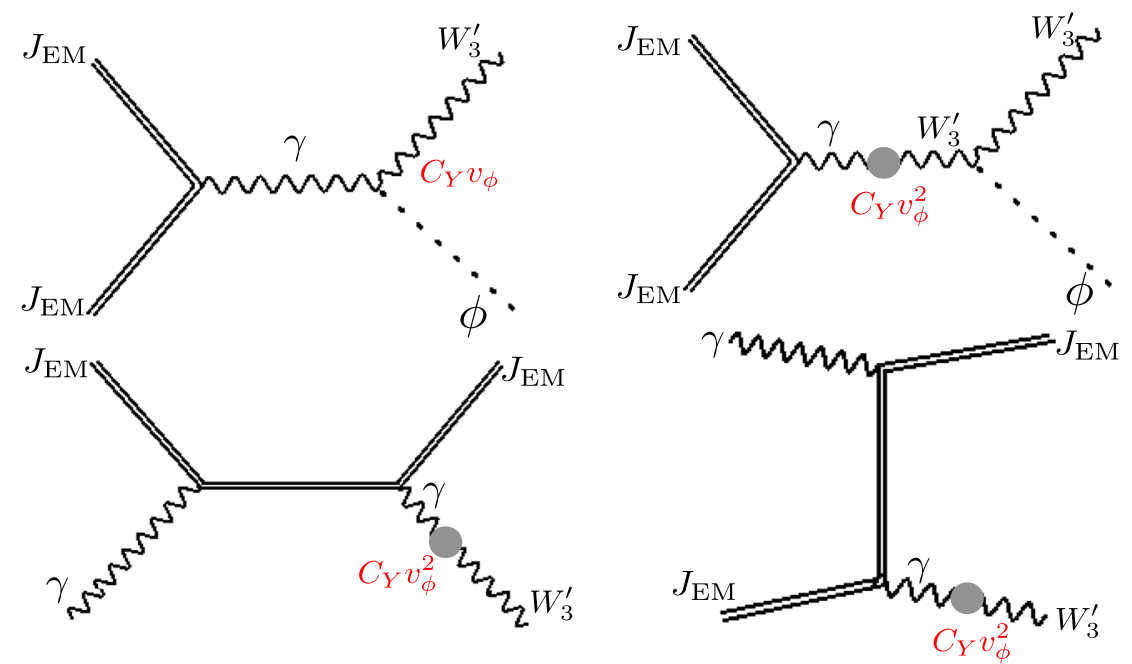

FIG. 6. The Feynman diagrams contributing to the production of $W_{3}^{\prime}$ and $\phi$ after $\phi$ acquires a vev. These diagrams are effective for $T<v_{\phi}$. In these diagrams, $J_{\mathrm{EM}}$ is any known $\mathrm{SM}$ particle that is still around at $T<v_{\phi}$. Note that since $v_{\phi} \simeq 60 \mathrm{MeV}$, the only electromagnetically charged particles that are still in the plasma and have not decayed are the electrons and protons.

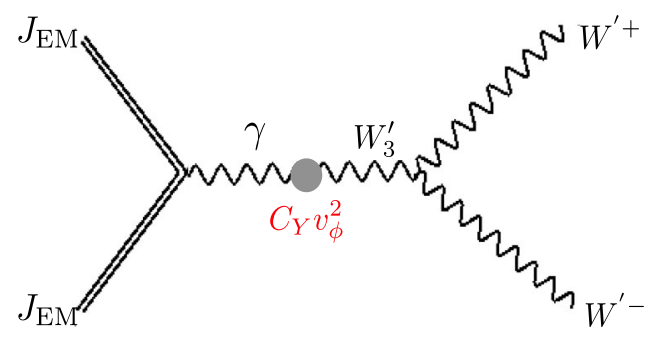

FIG. 7. The Feynman diagram that shows the production of $W^{\prime \pm}$ after $\phi$ acquires a vev.

these effects, we follow the convention of Ref. [75] and require $W_{3}^{\prime}$ to decay before it exceeds half of the energy density of the universe. The temperature at which this occurs is

$$
T_{\mathrm{dom}} \approx \frac{4 m_{W^{\prime}} Y_{W^{\prime}}}{3 f},
$$

where $f=1 / 2$ is the fraction of the energy density of the universe made up by $W^{\prime}$. We require that the lifetime of the $W_{3}^{\prime}$ is smaller than $H^{-1}\left(T_{\text {dom }}\right)$. The lifetime of $W_{3}^{\prime}$ if $m_{W^{\prime}}>2 m_{e}$ is

$$
\tau_{\text {heavy }} \simeq \frac{8 \pi}{\alpha C_{Y}^{2} v_{\phi} m_{W^{\prime}}}\left(1-\left(\frac{2 m_{e}}{m_{W^{\prime}}}\right)^{2}\right)^{-1 / 2}
$$

and, if it is lighter than $2 m_{e}$, is

$$
\tau_{\text {light }} \simeq \frac{2^{7} 3^{6} 5^{3} \pi^{3}}{17 C_{Y}^{2} v_{\phi}^{4} \alpha^{4} m_{e}}\left(\frac{m_{e}}{m_{W^{\prime}}}\right)^{9}
$$

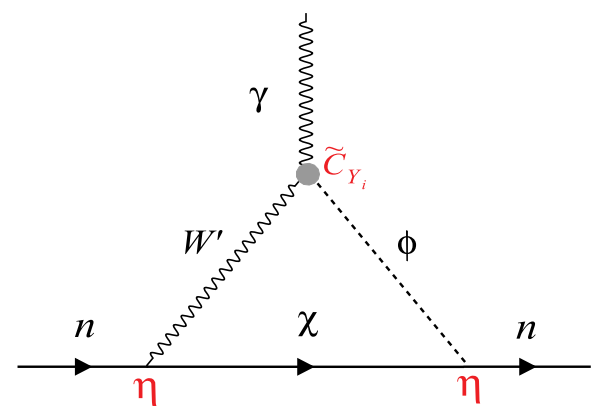

FIG. 8. The new contribution to the electric dipole moment of neutron. Since this diagram is suppressed by $\eta^{2}$, its contribution is very small.

As can be seen in Fig. 9 (shaded blue region), for $m_{W^{\prime}}<2 m_{e}$, this constraint is strongly restricting. However, for $m_{W^{\prime}}>2 m_{e}$, the BBN constraint becomes milder than the bound we get for the relic abundance.

\section{B. Indirect detection}

DM accumulating at the Galactic Center, or near dwarf spheroidal galaxies, annihilates to $W_{3}^{\prime}$ : (e.g., $W^{\prime+} W^{\prime-} \rightarrow$ $\left.W_{3}^{\prime(*)} W_{3}^{\prime}\right)$. Depending on the mass of $W_{3}^{\prime}$, we may either have $W_{3}^{\prime} \rightarrow e^{+} e^{-}$or $W_{3}^{\prime} \rightarrow 3 \gamma$. An excess emission of positron may be detected by Voyager [76] and the AMS-02 [77]. As discussed in [30,78], any claim on the detection of DM from the excess positron suffers from large uncertainties and it is not reliable.

The Fermi-LAT collaboration $[79,80]$ is searching for the excess in photons, and Ref. [30] has derived the constraint on DM coming from 6 years of FermiLAT observations of 15 dwarf spheroidal galaxies. 

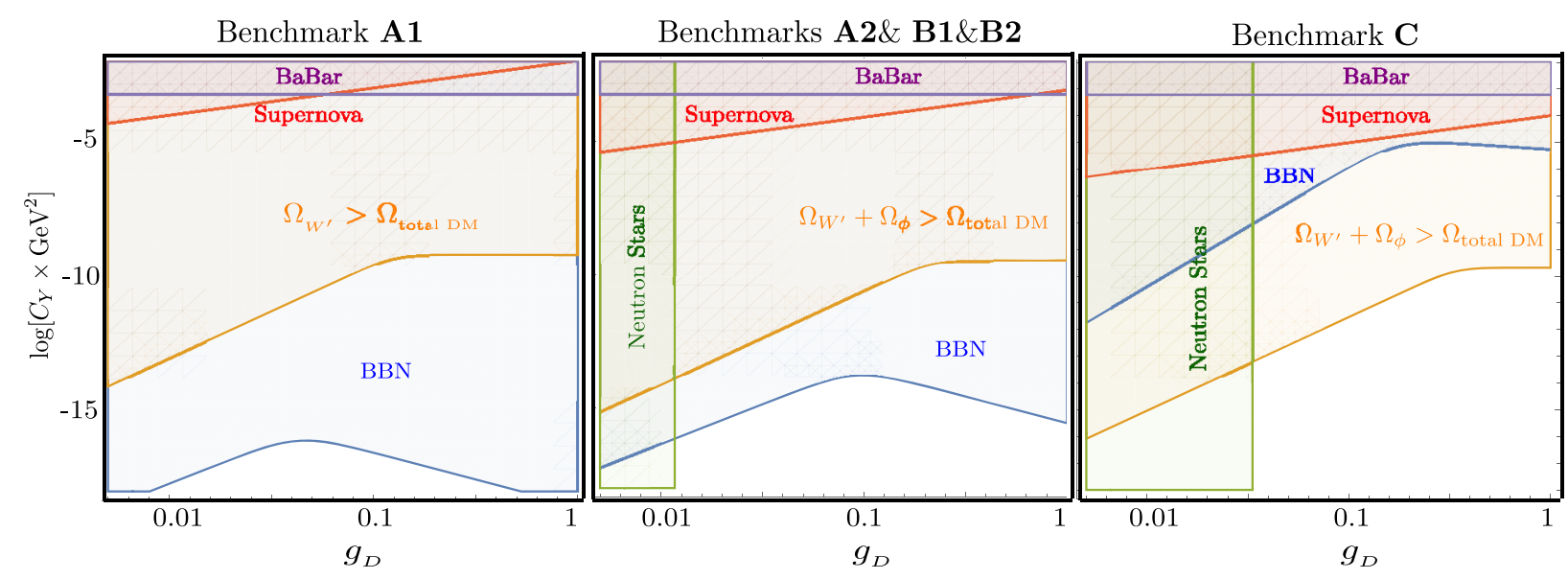

FIG. 9. These plots show the constraints from various experiments on different benchmarks. For the case where $m_{W^{\prime}}<2 m_{e}$, BBN [75] puts significant restrictions on the parameter space. For heavier $W^{\prime}$, the main constraints comes from Relic abundance (orange), and Neutron Stars (green) [30,51]. The bounds coming from SN1987A [85] and BABAR [83] are also shown in red and purple, respectively. The rest of the bounds discussed are much weaker than these bounds and are not presented here. The constraints are very sensitive to the value of $m_{W^{\prime}}$, but the value of $m_{\phi}$ does not visibly change the bounds. Thereby, we presented the bounds for benchmarks A2, B1, and B2 together.

Reference [30] has shown that the region parameter space satisfying $\langle\sigma v\rangle_{W^{\prime+} W^{\prime-} \rightarrow 3 \gamma W_{3}^{\prime} \rightarrow 6 \gamma}>2.8 \times 10^{-28} \mathrm{~cm}^{3} / \mathrm{s}$ is excluded. We can approximate this annihilation as

$$
\langle\sigma v\rangle_{W^{\prime+} W^{\prime-} \rightarrow 3 \gamma W_{3}^{\prime} \rightarrow 6 \gamma} \simeq \frac{\alpha_{D}^{2} C_{Y}^{2} v_{\phi}^{4}}{2^{8} 3^{6} 5^{3} \pi^{3} m_{W^{\prime}}^{2}},
$$

where $\alpha_{D}=g_{D}^{2} /(4 \pi)$. As can be seen, this cross section is extremely small and does not provide any noteworthy bound on the parameter space.

\section{Direct detection and collider constraints}

The cross section for $W^{\prime \pm}$ to scatter on proton with $W_{3}^{\prime}$ being the mediator is

$$
\sigma_{e W^{\prime}}=\frac{8 \pi \alpha \alpha_{D} C_{Y}^{2} v_{\phi}^{4} \mu_{e_{W^{\prime}}}^{2}}{\left(m_{W^{\prime}}^{2}+q^{2}\right)^{2}},
$$

where $\mu_{e_{W^{\prime}}} \equiv \frac{m_{W^{\prime}} m_{e}}{m_{W^{\prime}}+m_{e}}$ is the reduced mass of the DMelectron system, and $q$ is the momentum transfer between the DM and electron. At electron ionization experiments like SENSEI [81] and XENON10 [80], the targeted electrons are usually bound to atoms with typical velocity of a bound electron being $v_{e} \sim \alpha$. The minimum energy transferred required in these experiments to knock out the bound electron and detect the DM-electron scattering is $q \sim \alpha m_{e}$. For all of our benchmarks, we have $m_{W^{\prime}} \gg \alpha m_{e}$ and thus the momentum transfer can be neglected. For such heavy $W^{\prime}$, the bounds are rather very mild and they do not provide any noticeable bound on our parameter space.

\section{BABAR and SLAC}

Another constraint on $C_{Y}$ comes from the direct production of $W_{3}^{\prime}$ and $\gamma$ at E137 [82] and BABAR [83] experiments. Reference [30] has worked out this constraints and has found that $C_{Y}^{2} v_{\phi}^{4}<2.5 \times 10^{-8}$, which means that $C_{Y}<6 \times 10^{-4} \mathrm{GeV}^{-2}$ is a much weaker bound than the ones we have discussed so far. Since the coupling of $W_{3}^{\prime}$ with $W^{\prime \pm}$ does not play any role, this constraint is oblivious to the value of $g_{D}$. The $B A B A R$ bound is shown as shaded purple in Fig. 9.

Yet, another important constraint comes from the electron beam dump experiment at SLAC [84], which consists of a $20 \mathrm{GeV}$ electron beam hitting upon a set of fixed aluminum plates. Through the $W_{3}^{\prime}-\gamma$, we can create a pair of DM candidates $\left(W^{\prime \pm}\right): e N \rightarrow e N W_{3}^{\prime *} \rightarrow e N W^{\prime+} W^{\prime-}$. The DM would then travel through a $179 \mathrm{~m}$ hill, followed by $204 \mathrm{~m}$ of air, and then would be detected by an electromagnetic calorimeter. This process, however, is suppressed. That is because the production of on-shell $W_{3}^{\prime}$ is favored, which then would decay back to either $e^{+} e^{-}$ or $3 \gamma$. The large electron-positron pair background coming from the SM photon overwhelms the signal. The SLAC experiment requires $C_{Y}<0.5 \mathrm{GeV}^{-2}$.

\section{Electric dipole moment of neutrons}

Since we can introduce a $C P$-odd kinetic mixing between the non-Abelian fields strength and photon (e.g., $\tilde{C}_{Y} \phi^{\dagger} \tau^{a} \phi W_{a}^{\prime \mu \nu} F_{\mu \nu}$ ), we get a constraint on $\tilde{C}_{Y}$ from the contribution of this scenario on neutron Electric Dipole Moment (EDM). The leading contribution is shown in Fig. $8^{9}$ : Doing the calculation, we get

\footnotetext{
${ }^{9}$ If we had not turned off $\lambda_{\phi H}$ coupling, we could have an arguably more important contribution to neutron EDM.
} 


$$
d_{n}^{\text {new }} \simeq \frac{\tilde{C}_{Y i} v_{\phi} \eta^{2}}{8 \pi^{2}} \log \frac{\Lambda^{2}}{m_{W^{\prime}}^{2}}
$$

where $\Lambda^{2} \sim m_{\Phi_{1}} m_{\Phi_{2}}$. Measurements exclude any contribution to the electric dipole moment that exceeds $d_{n}^{\text {new }}<10^{-26} e . c m$. Given the value of $m_{W^{\prime}}$ in our benchmarks, EDM measurements require us to $\tilde{C}_{Y}<10^{12} \mathrm{GeV}^{-2}$, and this constraint is much weaker than perturbativity.

\section{Astrophysical bounds}

We have already summarized the importance of NS in constraining any model that discusses nonstandard neutron decay. Recall that to evade NS bounds, we moved to models with dark vector mediators, and we had to fix $m_{W^{\prime}} / g_{D} \sim 60 \mathrm{MeV}$. This constraint is presented in Fig. 9 as shaded green.

Another astrophysical bound comes from the cooling rate of Supernova1987A (SN1987A) [85]. Through the mixing with a photon, DM can be produced through the implosion of a newly born NS. Since DM does not interact with baryonic matter strongly, it can leave the supernova, resulting in a faster cooling rate. If DM is produced in an appreciable number, then the cooling rate can be faster than observed. For SN1987A, the energy loss per unit mass should be smaller than $10^{19} \mathrm{erg} / \mathrm{g} / \mathrm{s}$ at the temperature of the plasma, which equates roughly $10 \mathrm{MeV}$. The shaded red region in Fig. 9 illustrates the constraint coming from SN1987A.

\section{CONCLUSION}

In this paper, we presented a model that can explain the discrepancy between the total decay width of the neutron and its decay width to protons. In the Standard Model (SM), we expect the branching ratio of $n \rightarrow$ $p e^{-} \bar{v}_{e}$ to be $100 \%$. However, the two bottle experiment and beam experiment, which measured the decay width of the neutron, one by counting the remaining neutron and another by counting the produced protons, show a discrepancy in their results. One potential answer could be that neutrons decay to dark sector (DS) particles with a branching ratio of $1 \%$. The observation of heavy neutron stars with a mass of about two solar mass narrows our attention to DS models with vector mediators. This way, we ensure a repulsive interaction between dark matter (DM) candidates as well as between DM and neutrons. A thermal relic dark $U(1)_{D}$ gauge has already been discussed in detail and it has been that there is not available parameter space for $m_{A^{\prime}}<2 m_{e}$. We considered the production of DS through the freeze-in mechanism instead. Even with freeze-in, however, we showed that the region of the parameter space that explains the neutron decay anomaly, necessarily, leads to the overproduction of $\chi$-the fermionic DM candidate in our theory. Thereby, we considered a low $T_{\max }$ (e.g., $T_{\max } \sim m_{\chi}$ ). This temperature is valid according to the current constraints on the reheat temperature of the universe.

Since $\chi$ in this model cannot account for the relic abundance of DM in the early universe, we considered a DS with gauged $S U(2)_{D}$. The extra degrees of freedom in this model can successfully account for the observed relic abundance of DM. Yet, due to the intricate relationship between the particles of DS, the number of free parameters in this model is the same as the case of gauged $U(1)_{D}$.

One important advantage of DS scenarios that attempts to explain another theoretical or experimental anomaly is that the freedom over the new parameter space becomes much smaller. In this paper, we only had a few free parameters we could consider: the kinetic mixing coupling, the dark gauge coupling, and $m_{W^{\prime}}$. All of these parameters could vary over a small region. For $m_{W^{\prime}}>2 m_{e}$, satisfying the right relic abundance gave the best bound on the kinetic mixing between the two sectors. For lighter $W_{3}^{\prime}$ $\left(m_{W^{\prime}}<2 m_{e}\right)$, BBN constraints became much more significant. The main constraint on $g_{D}$ is from making sure the self-interaction of DM, as well as the interaction between DM and neutrons, are repulsive enough that they do not change the equation of state of large neutron stars significantly.

\section{ACKNOWLEDGMENTS}

We would like to thank H. Mehrabpour, D. McKeen, and J. Unwin for numerous useful conversations. F. E. is also thankful to CERN theory division and Mainz Cluster of Excellence for their hospitality.

\section{APPENDIX: THE SQUARED MATRIX ELEMENTS OF THE PROCESSES THAT PRODUCE $W^{\prime}$ AND $\phi$ FOR $T<v_{\phi}$.}

The exact Matrix Element of the processes presented in Figs. 6 and 7 are the following: 


$$
\begin{aligned}
|\mathcal{M}|_{J_{\mathrm{EM}} J_{\mathrm{EM}} \rightarrow \phi W^{\prime}}^{2}= & \frac{Q^{2} C_{Y}^{2} v_{\phi}^{2}}{4}\left(g_{D} v_{\phi}+\sqrt{s}\right)^{2} \frac{2 m_{W^{\prime}}^{2}\left(4 s^{2}+5 s(t+u)-5 t u\right)+5 s(s-t)^{2}}{m_{W^{\prime}}^{2} s^{2}} \\
|\mathcal{M}|_{\gamma_{J_{\mathrm{EM}} \rightarrow J_{\mathrm{EM}} W^{\prime}}}^{2}= & \frac{Q^{4} C_{Y}^{2} v_{\phi}^{4}}{4} \frac{\left(2 m_{W^{\prime}}^{2} s(2 s-t+u)+s^{2}(2 s-t-5 u)\right)}{m_{W^{\prime}}^{2}(s-t)^{2}} \\
|\mathcal{M}|_{J_{\mathrm{EM}} J_{\mathrm{EM}} \rightarrow W^{\prime+} W^{\prime-}}^{2}= & \frac{g_{D}^{2} Q^{2} C_{Y}^{2} v_{\phi}^{4}}{32 m_{W^{\prime}}^{4}\left(s-m_{W^{\prime}}^{2}\right.}\left(32 m_{\mathrm{SM}}^{4}\left(m_{W^{\prime}}^{2} s-4 m_{W^{\prime}}^{4}\right)\right. \\
& +2 m_{\mathrm{SM}}^{2}\left(s-4 m_{W^{\prime}}^{2}\right)\left(s^{2}+4 m_{W^{\prime}}^{2}(s+4(t+u))-20 m_{W^{\prime}}^{4}\right)-128 m_{W^{\prime}}^{8} \\
& -16 m_{W^{\prime}}^{6}(s-8(t+u))-4 m_{W^{\prime}}^{4}\left(s^{2}+8 s(t+u)+11 t^{2}+10 t u+11 u^{2}\right) \\
& \left.+4 m_{W^{\prime}}^{2} s\left(3 t^{2}+2 t u+3 u^{2}\right)+s^{2}\left(s^{2}-(t-u)^{2}\right)\right),
\end{aligned}
$$

where the Mandelstam $(s, t, u)$ variables are defined as usual:

$$
\begin{aligned}
& s=4\left(T^{2}+m_{\mathrm{SM}}^{2}\right) \\
& t=-2\left(T^{2}+m_{\mathrm{SM}}^{2}\right)+2 T \sqrt{T^{2}+m_{\mathrm{SM}}^{2}-m_{W^{\prime}}^{2}}(\cos \theta)+m_{\mathrm{SM}}^{2}+m_{W^{\prime}}^{2} \\
& u=-2\left(T^{2}+m_{\mathrm{SM}}^{2}\right)-2 T \sqrt{T^{2}+m_{\mathrm{SM}}^{2}-m_{W^{\prime}}^{2}}(\cos \theta)+m_{\mathrm{SM}}^{2}+m_{W^{\prime}}^{2} .
\end{aligned}
$$

In the limit where $s \gg t, u$ and $v_{\phi}>\sqrt{s}>m_{\mathrm{SM}}^{2}(T=0)$, we get

$$
\begin{aligned}
|\mathcal{M}|_{J_{\mathrm{EM}} J_{\mathrm{EM}} \rightarrow \phi W^{\prime}}^{2} & \simeq \frac{Q^{2} C_{Y}^{2} v_{\phi}^{2}\left(g_{D} v_{\phi}+\sqrt{s}\right)^{2} s}{m_{W^{\prime}}^{2}} \\
|\mathcal{M}|_{\gamma_{J_{\mathrm{EM}} \rightarrow J_{\mathrm{EM}} W^{\prime}}^{2}}^{2} & \simeq 2 \frac{Q^{4} C_{Y}^{2} v_{\phi}^{4} s}{m_{W^{\prime}}^{2}} \\
|\mathcal{M}|_{J_{\mathrm{EM}} J_{\mathrm{EM}} \rightarrow W^{\prime+} W^{\prime-}}^{2} & \simeq \frac{g_{D}^{2} Q^{2} C_{Y}^{2} v_{\phi}^{4} s^{2}}{8 m_{W^{\prime}}^{4}} .
\end{aligned}
$$

Given that for all of our benchmarks $v_{\phi}$ is at least an order of magnitude greater than $m_{W^{\prime}}$ and $m_{\phi}$, we can ignore $m_{\text {final }}$ in some of the cases of our interest. The yield, thus, becomes the following:

$$
\begin{aligned}
& Y^{T<v_{\phi}}=Y_{J_{\mathrm{EM}} J_{\mathrm{EM}} \rightarrow \phi W^{\prime}}+Y_{\gamma J_{\mathrm{EM}} \rightarrow J_{\mathrm{EM}} W^{\prime}}+Y_{J_{\mathrm{EM}} J_{\mathrm{EM}} \rightarrow W^{\prime} W^{\prime}} \\
& Y_{J_{\mathrm{EM}} J_{\mathrm{EM}} \rightarrow \phi W^{\prime}} \simeq g_{i} \alpha M_{\mathrm{Pl}} \frac{45 \times C_{Y}^{2}}{1.66 \times 16 \pi \sqrt{g_{\star}^{\rho}} g_{\star}^{S}} \frac{v_{\phi}^{5}}{m_{W^{\prime}}^{2}}\left[64 g_{D}\left(1-\frac{m_{\mathrm{final}}}{v_{\phi}}\right)+45 \pi\left(1-\left(\frac{m_{\mathrm{final}}}{v_{\phi}}\right)^{2}\right)\right]^{2}, \\
& Y_{\gamma J_{\mathrm{EM}} \rightarrow J_{\mathrm{EM}} W^{\prime}} \simeq g_{i} \alpha M_{\mathrm{Pl}} \frac{45 \times C_{Y}^{2}}{1.66 \times 4 \pi \sqrt{g_{\star}^{\rho}} g_{\star}^{S}} \frac{v_{\phi}^{5}}{m_{W^{\prime}}^{2}}\left[1-\frac{m_{\mathrm{final}}}{v_{\phi}}\right] \\
& Y_{J_{\mathrm{EM}} J_{\mathrm{EM}} \rightarrow W^{\prime} W^{\prime}} \simeq \alpha M_{\mathrm{Pl}} \frac{45 * 2^{5} \times C_{Y}^{2}}{1.66 \times \pi \sqrt{g_{\star}^{\rho}} g_{\star}^{S}} \frac{v_{\phi}^{7}}{m_{W^{\prime}}^{4}}\left[1-\left(\frac{m_{\mathrm{final}}}{v_{\phi}}\right)^{3}\right] .
\end{aligned}
$$


[1] W. Mampe, L. N. Bondarenko, V. I. Morozov, Yu. N. Panin, and A.I. Fomin, Measuring neutron lifetime by storing ultracold neutrons and detecting inelastically scattered neutrons, JETP Lett. 57, 82 (1993), http://www.jetpletters .ac.ru/ps/index-v-57_en.shtml.

[2] J. M. Robson, Radioactivity of the neutron (1951), pp. 143165.

[3] A. Serebrov et al., Measurement of the neutron lifetime using a gravitational trap and a low-temperature Fomblin coating, Phys. Lett. B 605, 72 (2005).

[4] A. Pichlmaier, V. Varlamov, K. Schreckenbach, and P. Geltenbort, Neutron lifetime measurement with the UCN trap-in-trap MAMBO II, Phys. Lett. B 693, 221 (2010).

[5] A. Steyerl, J. M. Pendlebury, C. Kaufman, S. S. Malik, and A. M. Desai, Quasielastic scattering in the interaction of ultracold neutrons with a liquid wall and application in a reanalysis of the Mambo I neutron-lifetime experiment, Phys. Rev. C 85, 065503 (2012).

[6] A. Czarnecki, W. J. Marciano, and A. Sirlin, Neutron Lifetime and Axial Coupling Connection, Phys. Rev. Lett. 120, 202002 (2018).

[7] A. T. Yue, M. S. Dewey, D. M. Gilliam, G. L. Greene, A. B. Laptev, J. S. Nico, W. M. Snow, and F. E. Wietfeldt, Improved Determination of the Neutron Lifetime, Phys. Rev. Lett. 111, 222501 (2013).

[8] F. E. Wietfeldt and G. L. Greene, Colloquium: The neutron lifetime, Rev. Mod. Phys. 83, 1173 (2011).

[9] J. Byrne et al., Measurement of the Neutron Lifetime by Counting Trapped Protons, Phys. Rev. Lett. 65, 289 (1990).

[10] A. T. Yue, M. S. Dewey, D. M. Gilliam, G. L. Greene, A. B. Laptev, J. S. Nico, W. M. Snow, and F. E. Wietfeldt, Improved Determination of the Neutron Lifetime, Phys. Rev. Lett. 111, 222501 (2013).

[11] David McKeen and Maxim Pospelov, How long does the hydrogen atom live?, arXiv:2003.02270.

[12] M. Agostini et al. (Borexino Collaboration), A Test of Electric Charge Conservation with Borexino, Phys. Rev. Lett. 115, 231802 (2015).

[13] K. Abe et al. (Super-Kamiokande Collaboration), Search for proton decay via $p \rightarrow e^{+} \pi^{0}$ and $p \rightarrow \mu^{+} \pi^{0}$ in 0.31 megaton years exposure of the Super-Kamiokande water Cherenkov detector, Phys. Rev. D 95, 012004 (2017).

[14] D. G. Phillips II et al., Neutron-antineutron oscillations: Theoretical status and experimental prospects, Phys. Rep. 612, 1 (2016).

[15] I. Goldman, R. N. Mohapatra, and S. Nussinov, Bounds on neutron-mirror neutron mixing from pulsar timing, Phys. Rev. D 100, 123021 (2019).

[16] Y. Grossman, W. H. Ng, and S. Ray, Revisiting the bounds on hydrogen-antihydrogen oscillations from diffuse $\gamma$-ray surveys, Phys. Rev. D 98, 035020 (2018).

[17] G. K. Leontaris and J. D. Vergados, $n-\bar{n}$ oscillations and the neutron lifetime, Phys. Rev. D 99, 015010 (2019).

[18] Z. Berezhiani, R. Biondi, P. Geltenbort, I. A. Krasnoshchekova, V. E. Varlamov, A. V. Vassiljev, and O. M. Zherebtsov, New experimental limits on neutron-mirror neutron oscillations in the presence of mirror magnetic field, Eur. Phys. J. C 78, 717 (2018).
[19] S. Gardner and X. Yan, Phenomenology of neutronantineutron conversion, Phys. Rev. D 97, 056008 (2018).

[20] R. Aaij et al. (LHCb Collaboration), Search for BaryonNumber Violating $\Xi_{b}^{0}$ Oscillations, Phys. Rev. Lett. 119, 181807 (2017).

[21] B. Aharmim et al. (SNO Collaboration), Search for neutronantineutron oscillations at the Sudbury neutrino observatory, Phys. Rev. D 96, 092005 (2017).

[22] A. K. Fomin, A. P. Serebrov, O. M. Zherebtsov, E. N. Leonova, and M.E. Chaikovskii, Experiment on search for neutron-antineutron oscillations using a projected UCN source at the WWR-M reactor, J. Phys. Conf. Ser. 798, 012115 (2017).

[23] J.E. T. Hewes, Searches for bound neutron-antineutron oscillation in liquid argon time projection chambers, $\mathrm{Ph}$. D. thesis, Manchester U., 2017.

[24] Y.-F. Liu and X.-W. Kang, Test baryon antibaryon oscillation in collider experiments, J. Phys. Conf. Ser. 738, 012043 (2016).

[25] M. J. Frost (NNbar), The NNbar experiment at the european spallation source, in 7th Meeting on CPT and Lorentz. Symmetry, Bloomington, Indiana, USA, (World Scientific, Singapore, 2017), pp. 265-267.

[26] K. Aitken, D. McKeen, T. Neder, and A. E. Nelson, Baryogenesis from oscillations of charmed or beautiful baryons, Phys. Rev. D 96, 075009 (2017).

[27] D. McKeen and A. E. Nelson, CP violating baryon oscillations, Phys. Rev. D 94, 076002 (2016).

[28] B. Fornal and B. Grinstein, Dark Matter Interpretation of the Neutron Decay Anomaly, Phys. Rev. Lett. 120, 191801 (2018).

[29] H. Davoudiasl, Nucleon Decay into a Dark Sector, Phys. Rev. Lett. 114, 051802 (2015).

[30] J. M. Cline and J. M. Cornell, Dark decay of the neutron, J. High Energy Phys. 07 (2018) 081.

[31] D. Barducci, M. Fabbrichesi, and E. Gabrielli, Neutral hadrons disappearing into the darkness, Phys. Rev. D 98, 035049 (2018).

[32] A. N. Ivanov, R. Hllwieser, N. I. Troitskaya, M. Wellenzohn, and Ya A. Berdnikov, Neutron dark matter decays and correlation coefficients of neutron $\beta^{-}$-decays, Nucl. Phys. B938, 114 (2019).

[33] B. Fornal and B. Grinstein, Neutron lifetime discrepancy as a sign of a dark sector?, Report No. CIPANP2018-Fornal (2018), arXiv:1810.00862.

[34] T. Bringmann, J. M. Cline, and J. M. Cornell, Baryogenesis from neutron-dark matter oscillations, Phys. Rev. D 99, 035024 (2019).

[35] B. Fornal and B. Grinstein, Dark side of the neutron?, EPJ Web Conf. 219, 05005 (2019).

[36] B. Grinstein, C. Kouvaris, and N. G. Nielsen, Neutron Star Stability in Light of the Neutron Decay Anomaly, Phys. Rev. Lett. 123, 091601 (2019).

[37] Z. Berezhiani, Neutron lifetime and dark decay of the neutron and hydrogen, Lett. High Energy Phys. 2, 118 (2019).

[38] Z. Berezhiani, Neutron lifetime puzzle and neutron-mirror neutron oscillation, Eur. Phys. J. C 79, 484 (2019). 
[39] M. Fabbrichesi and A. Urbano, Charged neutron stars and observational tests of a dark force weaker than gravity, J. Cosmol. Astropart. Phys. 06 (2020) 007.

[40] B. Fornal and B. Grinstein, Dark particle interpretation of the neutron decay anomaly, J. Phys. Conf. Ser. 1308, 012010 (2019).

[41] R. Garani, Y. Genolini, and T. Hambye, New analysis of neutron star constraints on asymmetric dark matter, J. Cosmol. Astropart. Phys. 05 (2019) 035.

[42] W.-Y. Keung, D. Marfatia, and P.-Y. Tseng, Heating neutron stars with GeV dark matter, arXiv:2001.09140.

[43] F. E. Wietfeldt et al., A Comment on "The possible explanation of neutron lifetime beam anomaly" by A. P. Serebrov et al., arXiv:2004.01165.

[44] D. Dubbers, H. Saul, B. Mrkisch, T. Soldner, and H. Abele, Exotic decay channels are not the cause of the neutron lifetime anomaly, Phys. Lett. B 791, 6 (2019).

[45] F. E. Wietfeldt, Measurements of the neutron lifetime, Atoms 6, 70 (2018).

[46] S. I. Blinnikov, Mirror matter and other dark matter models, Usp. Fiz. Nauk 184, 194 (2014).

[47] S. I. Blinnikov, Mirror matter and other models for dark matter, in 100th Anniversary of the Birth of I. Ya. Pomeranchuk, Moscow, Russia (2014), Vol. 57, pp. 152-208.

[48] M. Khlopov, Cosmoparticle physics of dark matter, EPJ Web Conf. 222, 01006 (2019).

[49] Z. Tang et al., Search for the Neutron Decay $\mathrm{n} \rightarrow \mathrm{X}+\gamma$ where X is a Dark Matter Particle, Phys. Rev. Lett. 121, 022505 (2018).

[50] M. Klopf, E. Jericha, B. Mrkisch, H. Saul, T. Soldner, and H. Abele, Constraints on the Dark Matter Interpretation $n \rightarrow \chi+e^{+} e^{-}$of the Neutron Decay Anomaly with the PERKEO II experiment, Phys. Rev. Lett. 122, 222503 (2019).

[51] D. McKeen, A. E. Nelson, S. Reddy, and D. Zhou, Neutron Stars Exclude Light Dark Baryons, Phys. Rev. Lett. 121, 061802 (2018).

[52] T. F. Motta, P. A. M. Guichon, and A. W. Thomas, Implications of neutron star properties for the existence of light dark matter, J. Phys. G 45, 05LT01 (2018).

[53] R. C. Tolman, Static solutions of Einstein's field equations for spheres of fluid, Phys. Rev. 55, 364 (1939).

[54] J. R. Oppenheimer and G. M. Volkoff, On massive neutron cores, Phys. Rev. 55, 374 (1939).

[55] P. Demorest, T. Pennucci, S. Ransom, M. Roberts, and J. Hessels, Shapiro delay measurement of a two solar mass neutron star, Nature (London) 467, 1081 (2010).

[56] S. Gandolfi, J. Carlson, and S. Reddy, The maximum mass and radius of neutron stars and the nuclear symmetry energy, Phys. Rev. C 85, 032801 (2012).

[57] Y. Aoki, T. Izubuchi, E. Shintani, and A. Soni, Improved lattice computation of proton decay matrix elements, Phys. Rev. D 96, 014506 (2017).

[58] A. M Sirunyan et al. (CMS Collaboration), Search for new physics with dijet angular distributions in proton-proton collisions at $\sqrt{s}=13 \mathrm{TeV}$, J. High Energy Phys. 07 (2017) 013.

[59] M. Aaboud et al. (ATLAS Collaboration), Search for squarks and gluinos in final states with jets and missing transverse momentum using $36 \mathrm{fb}^{-1}$ of $\sqrt{s}=13 \mathrm{TeV}$ pp collision data with the ATLAS detector, Phys. Rev. D 97, 112001 (2018).

[60] J. M. Cline and P. Scott, Dark Matter CMB Constraints and Likelihoods for Poor Particle Physicists, J. Cosmol. Astropart. Phys. 03 (2013) 044; Erratum: J. Cosmol. Astropart. Phys. 05 (2013) E01.

[61] S. Tulin and H.-B. Yu, Dark matter self-interactions and small scale structure, Phys. Rep. 730, 1 (2018).

[62] G. K. Karananas and A. Kassiteridis, Small-scale structure from neutron dark decay, J. Cosmol. Astropart. Phys. 09 (2018) 036.

[63] M. Hufnagel, K. Schmidt-Hoberg, and S. Wild, BBN constraints on MeV-scale dark sectors. Part I. Sterile decays, J. Cosmol. Astropart. Phys. 02 (2018) 044.

[64] A. Drlica-Wagner et al. (Fermi-LAT and DES Collaborations), Search for gamma-ray emission from DES Dwarf spheroidal galaxy candidates with Fermi-LAT data, Astrophys. J. 809, L4 (2015).

[65] S. W. Randall, M. Markevitch, D. Clowe, A. H. Gonzalez, and M. Bradac, Constraints on the self-interaction crosssection of dark matter from numerical simulations of the merging galaxy cluster 1E 0657-56, Astrophys. J. 679, 1173 (2008).

[66] L. J. Hall, K. Jedamzik, J. March-Russell, and S. M. West, Freeze-in production of FIMP dark matter, J. High Energy Phys. 03 (2010) 080.

[67] F. Elahi, C. Kolda, and J. Unwin, UltraViolet freeze-in, J. High Energy Phys. 03 (2015) 048.

[68] M. A. G. Garcia, Y. Mambrini, K. A. Olive, and M. Peloso, Enhancement of the dark matter abundance before reheating: Applications to gravitino dark matter, Phys. Rev. D 96, 103510 (2017).

[69] K. Kaneta, Y. Mambrini, and K. A. Olive, Radiative production of nonthermal dark matter, Phys. Rev. D 99, 063508 (2019).

[70] N. Bernal, M. Dutra, Y. Mambrini, K. Olive, M. Peloso, and M. Pierre, Spin-2 portal dark matter, Phys. Rev. D 97, 115020 (2018).

[71] D. Chowdhury, E. Dudas, M. Dutra, and Y. Mambrini, Moduli Portal Dark Matter, Phys. Rev. D 99, 095028 (2019).

[72] N. Bernal, F. Elahi, C. Maldonado, and J. Unwin, Ultraviolet freeze-in and non-standard cosmologies, J. Cosmol. Astropart. Phys. 11 (2019) 026.

[73] A. Czarnecki, W. J. Marciano, and A. Sirlin, Neutron Lifetime and Axial Coupling Connection, Phys. Rev. Lett. 120, 202002 (2018).

[74] G. F. Giudice, E. W. Kolb, and A. Riotto, Largest temperature of the radiation era and its cosmological implications, Phys. Rev. D 64, 023508 (2001).

[75] M. Cirelli, P. Panci, K. Petraki, F. Sala, and M. Taoso, Dark Matter's secret liaisons: Phenomenology of a dark U(1) sector with bound states, J. Cosmol. Astropart. Phys. 05 (2017) 036.

[76] E. C. Stone, A. C. Cummings, F. B. McDonald, B. C. Heikkila, N. Lal, and W. R. Webber, Voyager 1 observes low-energy galactic cosmic rays in a region depleted of heliospheric ions, Science 341, 150 (2013).

[77] P. A. R. Ade et al. (POLARBEAR Collaboration), Measurement of the Cosmic Microwave Background Polarization 
Lensing Power Spectrum with the Polarbear Experiment, Phys. Rev. Lett. 113, 021301 (2014).

[78] M. Boudaud, J. Lavalle, and P. Salati, Novel Cosmic-Ray Electron and Positron Constraints on MeV Dark Matter Particles, Phys. Rev. Lett. 119, 021103 (2017).

[79] M. Ackermann et al. (Fermi-LAT Collaboration), Searching for Dark Matter Annihilation from Milky Way Dwarf Spheroidal Galaxies with Six Years of Fermi Large Area Telescope Data, Phys. Rev. Lett. 115, 231301 (2015).

[80] S. Bergstrm et al., J-factors for self-interacting dark matter in 20 dwarf spheroidal galaxies, Phys. Rev. D 98, 043017 (2018).

[81] M. Crisler, R. Essig, J. Estrada, G. Fernandez, J. Tiffenberg, M. Sofo haro, T. Volansky, and T.-T. Yu (SENSEI Collaboration), SENSEI: First Direct-Detection Constraints on sub-GeV Dark Matter from a Surface Run, Phys. Rev. Lett. 121, 061803 (2018).
[82] S. Andreas, C. Niebuhr, and A. Ringwald, New limits on hidden photons from past electron beam dumps, Phys. Rev. D 86, 095019 (2012).

[83] J. P. Lees et al. (BABAR Collaboration), Search for Invisible Decays of a Dark Photon Produced in $e^{+} e^{-}$Collisions at BABAR, Phys. Rev. Lett. 119, 131804 (2017).

[84] A. A. Prinz, R. Baggs, J. Ballam, S. Ecklund, C. Fertig, J. A. Jaros, K. Kase, A. Kulikov, W. G. J. Langeveld, R. Leonard, T. Marvin, T. Nakashima, W. R. Nelson, A. Odian, M. Pertsova, G. Putallaz, and A. Weinstein, Search for Millicharged Particles at SLAC, Phys. Rev. Lett. 81, 1175 (1998).

[85] J. H. Chang, R. Essig, and S. D. McDermott, Supernova 1987A constraints on sub-GeV dark sectors, millicharged particles, the QCD axion, and an axion-like particle, J. High Energy Phys. 09 (2018) 051. 